\title{
Variations in injury characteristics among paediatric patients following trauma: A retrospective descriptive analysis comparing pre-hospital and in-hospital deaths at Kamuzu Central Hospital, Lilongwe, Malawi
}

\author{
Laura Purcell $^{1}$, Charles E. Mabedii, ${ }^{2,3}$, Jared Gallaher ${ }^{1}$, Steven Mjuweni ${ }^{2}$, Sean McLean ${ }^{1}$, Bruce Cairns $^{1}$, \\ Anthony Charles ${ }^{1}$
}

1. Department of Surgery, University of North Carolina, Chapel Hill, North Carolina, USA

2. Department of Surgery, Kamuzu Central Hospital, Lilongwe, Malawi

3. Malawi Surgical Initiative, UNC Project-Malawi, Lilongwe, Malawi

Correspondence: Dr Anthony Charles (anthchar@med.unc.edu)

\begin{abstract}
Background
Abstract

Trauma is a major cause of paediatric mortality in sub-Saharan Africa. In absence of pre-hospital care, the injury mechanism and cause of death is difficult to characterise. Injury characteristics of pre-hospital deaths (PHD) versus in-hospital deaths (IHD) were compared. Methods

Using our trauma surveillance database, a retrospective, descriptive analysis of children ( $<18$ years) presenting to Kamuzu Central Hospital in Lilongwe, Malawi from 2008 to 2013 was performed. Patient and injury characteristics of pre-hospital and in-hospital deaths were compared with univariate and bivariate analysis.
\end{abstract}

Results

Of 30,462 paediatric trauma patients presenting between 2008 and 2013, 170 and 173 were PHD and IHD, respectively. In PHD and IHD patients mean age was $7.3 \pm 4.9$ v $5.2 \pm 4.3(\mathrm{p}<0.001)$, respectively. IHD patients were more likely transported via ambulance than those PHD, $51.2 \%$ v $8.3 \%$ ( $<<0.001)$. The primary mechanisms of injury for PHD were road traffic injuries (RTI) (45.8\%) and drowning $(22.0 \%)$, with head injury (46.7\%) being the predominant cause of death. Burns were the leading mechanism of injury (61.8\%) and cause of death $(61.9 \%)$ in IHD, with a mean total body surface area involvement of $24.7 \pm 16.0 \%$.

Conclusions

RTI remains Malawi's major driver of paediatric mortality. A majority of these deaths attributed to head injury occur prior to hospitalisation; therefore the mortality burden is underestimated if accounting for IHD alone. Death in burn patients is likely due to under-resuscitation or sepsis. Improving pre-hospital care and head injury and burn management can improve injury related paediatric mortality.

\section{Introduction}

Traumatic injury is a major cause of premature death and disability worldwide and remains the leading cause of death in the first four decades of life. Injury accounts for up to $16 \%$ of the global burden of disease, with road traffic injury being the major driver of traumatic injuries. Unfortunately low and middle-income countries (LMICs) bare a disproportionate burden of trauma morbidity and mortality, where $90 \%$ of all trauma-related deaths and $95 \%$ of paediatric injuries occur. Of the world's regions, sub-Saharan Africa has suffered the most. The World Health Organization (WHO) and United Nations Children's Emergency Fund (UNICEF) considers paediatric trauma a major health concern requiring urgent attention in their World Report on Child Injury Prevention in 2008 .

The management of trauma victims, either from road traffic injuries (RTI), falls, civil strife, or wars, requires functional trauma care systems in any global setting. One of the hallmarks of a mature trauma system is the robustness of the pre-hospital care delivery at the point of injury. In the United States, several studies have shown that the implementation of a trauma system extending from pre-hospital care delivery to in-hospital care and on through rehabilitation and discharge, lowers trauma related mortality and morbidity., Unfortunately, most countries in sub-Saharan Africa lack an organised trauma system, especially a pre-hospital care delivery system.

There is also a paucity of data regarding trauma outcomes in LMIC's, especially regarding pre-hospital deaths, as most LMIC's also lack a trauma surveillance registry.4 In order to better ascertain the characteristics of paediatric trauma in sub-Saharan Africa, we examined the variations in fatal injury in paediatric trauma patients evaluating pre-hospital and in-hospital mortality data in Malawi.

\section{Methods}

We conducted a retrospective descriptive analysis of a hospital-based paediatric trauma surveillance registry from 2008 - 2013 at Kamuzu Central Hospital $(\mathrm{KCH})$ in Lilongwe, Malawi. All patients that present to the emergency department with traumatic injuries are recorded. Paediatric patients less than 18 years old were included in the analysis. Less than 18 years of age was selected, as this is the generally accepted legal and global standard definition for children.

$\mathrm{KCH}$ is a 600-bed hospital and a referral center for the central region of the country, with a population of about five million persons. There are dedicated children's wards including: a surgical ward, paediatric high dependency unit, and intermediate care ward. During the study period, there were four full-time general surgeons, three full-time orthopaedic 
surgeons, multiple clinical officers, and an 11 resident general surgery residency-training program. In addition, there is a 31-bed burn unit. There is no pre-hospital care system in Malawi and minimal basic life support measures are available prior to arrival at the hospital.

The primary outcome was death, categorised as either prehospital death (PHD) or in-hospital death (IHD). PHD is defined as deaths occurring prior to arrival to $\mathrm{KCH}$. IHD is defined as deaths occurring at $\mathrm{KCH}$, either in the emergency department or death after admission to the hospital. Data collected included basic demographics, injury mechanism, mode of transportation to the hospital, and clinical examination findings to determine injury characteristics.

We initially examined the characteristics of the sample to assess the distribution of the variables, as well as to assess any impact of missing data or extreme values. The mean, standard deviation, and the shape of the distribution were calculated for each continuous variable, and frequencies were tabulated for categorical variables. We compared demographic and clinical variables between the PHD and IHD cohorts. For categorical variables, we used a Pearson's Chi square test and for continuous variables, a Student's twosample T-test. When comparing categorical variables with more than two categories, the aggregate of the remaining categories was used as the referent for comparison.

Data analysis was performed using STATA v14.1. The institutional review board at the University of North Carolina at Chapel Hill and the National Health Science and Research Council of Malawi approved this study.

\section{Results}

A total of 30,462 children with traumatic injury presented to the $\mathrm{KCH}$ during the study period and 5,024 children were admitted. 170 children died at the scene or in transport and were dead prior to being assessed at the hospital (PHD). Inhospital deaths included 173 children who died while in the emergency department or after being admitted to $\mathrm{KCH}$. The PHD cohort had a mean age of $7.3 \pm 4.9$ years compared to the IHD with a mean age of $5.2 \pm 4.3$ years $(p<0.001)$. Most deaths occurred in the 0-6 years age group with $47.7 \%$ and $72.8 \%$ in the PHD and IHD groups, respectively. Similarly, males suffered the majority of deaths for both PHD and IHD of $60.0 \%$ and $55.2 \%$, respectively $(p=0.256)$.

In the PHD cohort, the predominant injury mechanism was RTI, which included pedestrian versus vehicle $(37.5 \%, \mathrm{n}=$ 63) and motor vehicle collision $(8.3 \%, \mathrm{n}=14)$. Drowning $(22.0 \%, \mathrm{n}=37)$, burns $(11.3 \%, \mathrm{n}=19)$, and assault $(9.5 \%, \mathrm{n}$ $=16)$ followed road traffic accidents as leading mechanisms of injury. In the IHD cohort, the most common mechanism of injury was burns $(61.8 \%, \mathrm{n}=107)$. The mean total body surface area involvement was $24.5 \pm 16.0 \%$. The mechanism was primarily scald burns $(57.7 \%, \mathrm{n}=60)$ versus flame burns $(39.4 \%, \mathrm{n}=41)$. The second leading injury mechanism was road traffic injuries, with pedestrian versus vehicle $(13.9 \%$, $\mathrm{n}=24)$ and motor vehicle collision $(7.5 \%, \mathrm{n}=13)$. Other injury mechanisms included falls $(4.6 \%, \mathrm{n}=8)$, injury from collapsed structure $(5.8 \%, \mathrm{n}=10)$, and assault $(2.9 \%, \mathrm{n}=5)$ ( $\mathrm{p}<0.001$, comparing PHD vs IHD).

In the PHD cohort, the predominant primary injury causing death is head injuries $(46.7 \%, \mathrm{n}=77)$, followed by burns $(7.3 \%, \mathrm{n}=12)$, and soft tissue injury $(6.1 \%, \mathrm{n}=10)$. The injuries in the IHD cohort were predominantly from burns $(61.9 \%, \mathrm{n}=107)$. Other significant primary injuries in IHD were head injures $(22.5 \%, \mathrm{n}=39)$ and long bone fractures $(5.8 \%, \mathrm{n}=10)$.

http://dx.doi.org/10.4314/mmj.v29i2.13
There were significant differences in the mode of transportation from scene of injury to the emergency department in both the PHD and IHD groups. Most patients in the PHD group presented by private vehicle $(50.6 \%, \mathrm{n}=$ 85 ) or police $(30.4 \%, \mathrm{n}=51)$, compared to IHD patients who presented to the hospital by ambulance $(51.2 \%, \mathrm{n}=$ $88)$ and private vehicle $(28.5 \%, \mathrm{n}=49)$. A small proportion of patients also presented via public transportation (minibus) at $9.5 \%$ of PHD $(n=16)$ and $18.6 \%(n=32)$ of IHD. Combining both PHD and IHD groups, 18.8\% (52 of 276) of patients transported by police died pre- or inhospital, compared to $2.6 \%$ (102 of 3,915) of transported via ambulance and 1.8\% (134 of 7,450) of transported by private vehicle $(\mathrm{p}<0.001)$.

The mean time from injury to hospital presentation for PHD patients was $0.3 \pm 1.0$ hour with $86.2 \%$ arriving in the first 4 hours $(n=85)$. IHD presented with a mean of $1.60 \pm 2.0$ hours $(\mathrm{p}<0.001)$. The majority of IHD arrived in less than four hours $(47.4 \%, \mathrm{n}=81)$, but with a significant number of patients arriving over 24 hours after injury $(22.2 \%, \mathrm{n}=$ 38). Time to mortality for IHD patients was $2.2 \pm 1.9$ days. Isolating IHD burn trauma resulted in an average time to mortality of $12.1 \pm 13.7$ days.

As the PHD mechanism of injury was more RTI related, deaths in the PHD cohort were more likely to occur on roads $(48.1 \%, \mathrm{n}=77)$, compared to $\operatorname{IHD}(21.5 \%, \mathrm{n}=37)(\mathrm{p}$ $<0.001)$. The majority of IHD occurred at home $(72.1 \% \mathrm{n}$ $=124)$, contrasted with significantly less PHD $(36.3 \%, \mathrm{n}=$ 58) $(\mathrm{p}<0.001)$

\section{Discussion}

This study is an analysis of two distinct paediatric trauma cohorts of pre-hospital and in-hospital deaths in a subSaharan African setting. Most importantly, the mechanism of injury in the PHD cohort was predominantly related to RTI, in contrast to the IHD cohort, which was primarily burn injury. In addition, the primary cause of death following paediatric trauma was head injury.

Sub-Saharan Africa demonstrates a higher head injuryrelated incidence rate varying from 150 to 170 per 100,000 due to RTI compared to a global rate of 106 per 100,000. This is due to rapid urbanisation with shared use of roads by motor vehicles, bicyclists, pedestrians, and other modes of transportation. This is combined with paucity of traffic rules and regulations, for example many open trucks passengers are unseated and safety belts are rarely used. Therefore a RTI in sub-Saharan Africa is more likely to involve a vehicle versus pedestrian or vehicle versus bicyclist than it is to involve vehicle versus vehicle. Paediatric patients are at higher risk of head trauma due to their larger and heavier cranial vault, which results in higher degrees of torque, and decreased myelination.

Our study reveals the consequences of a lack of a formalised trauma system. In Malawi, police, minibus and taxi drivers, or Good Samaritans provide pre-hospital care on a voluntary basis after road-related emergencies. By analyzing only inhospital data, the aetiology of paediatric trauma mortality would severely underrepresent all cause trauma mortality. For example, a study performed in Ghana showed only $31 \%$ of fatal injuries received formal medical treatment. Similar data from the United States indicated $78 \%$ of paediatric trauma deaths were missed by only examining in-hospital data. An analysis of pre-hospital and in-hospital mortality must be 
Table 1: Bivariate analysis of demographic and clinical characteristics of paediatric patients presenting with traumatic injuries, comparing pre-hospital deaths with in-hospital deaths

\begin{tabular}{|c|c|c|c|}
\hline Variable & $\begin{array}{l}\text { Pre-hospital deaths } \\
\qquad(\mathrm{n}=170)\end{array}$ & $\begin{array}{c}\text { In-hospital deaths } \\
\qquad(\mathrm{n}=173)\end{array}$ & P-value \\
\hline \multicolumn{4}{|l|}{ Patient age (years) } \\
\hline Mean \pm standard deviation & $7.3 \pm 4.9$ & $4.24 \pm 4.34$ & $<0.001$ \\
\hline \multicolumn{4}{|l|}{ Gender, n (\%) } \\
\hline Male & $103(60.6)$ & $95(55.2)$ & \multirow{3}{*}{0.256} \\
\hline Female & $65(38.2)$ & $77(44.8)$ & \\
\hline Missing & 2 & 1 & \\
\hline \multicolumn{4}{|l|}{ Mechanism of injury, n (\%) } \\
\hline Vehicular & $77(45.8)$ & $37(21.4)$ & \multirow{10}{*}{$<0.001$} \\
\hline Burn & $19(11.3)$ & $107(61.8)$ & \\
\hline Drowning & $37(22.0)$ & $2(1.2)$ & \\
\hline Assault & $16(9.5)$ & $5(2.9)$ & \\
\hline Collapsed structure & $11(6.5)$ & $10(5.8)$ & \\
\hline Fall & $5(3.0)$ & $8(4.6)$ & \\
\hline Gunshot wound & $3(1.8)$ & 0 & \\
\hline Foreign body & 0 & $3(1.7)$ & \\
\hline Other & $3(1.8)$ & $1(0.6)$ & \\
\hline Missing & 2 & 0 & \\
\hline \multicolumn{4}{|l|}{ Injury pattern, $\mathbf{n}(\%)$} \\
\hline Head & $77(46.7)$ & $39(22.5)$ & \multirow{7}{*}{$<0.001$} \\
\hline Burn & $12(7.3)$ & 107 (61.9) & \\
\hline Soft tissue & $10(6.1)$ & $5(2.9)$ & \\
\hline Fracture & $8(4.9)$ & $10(5.8)$ & \\
\hline Penetrating & $7(4.2)$ & $1(0.6)$ & \\
\hline Other & $51(30.9)$ & $11(6.4)$ & \\
\hline Missing & 5 & 0 & \\
\hline \multicolumn{4}{|l|}{ Injury location, n (\%) } \\
\hline Head/face & $89(71.8)$ & $65(37.8)$ & \multirow{9}{*}{$<0.001$} \\
\hline Abdomen/flank & $14(11.3)$ & $36(20.9)$ & \\
\hline Upper extremity & $7(5.7)$ & $8(4.7)$ & \\
\hline Chest & $6(4.8)$ & $14(8.1)$ & \\
\hline Lower extremity & $4(3.2)$ & $15(8.7)$ & \\
\hline Hand & $2(1.6)$ & $11(6.4)$ & \\
\hline Pelvis & $1(0.8)$ & $7(4.1)$ & \\
\hline Spine & $1(0.8)$ & $16(9.3)$ & \\
\hline Missing & 0 & 1 & \\
\hline \multicolumn{4}{|l|}{ Transportation, n (\%) } \\
\hline Private vehicle & $85(50.6)$ & $49(28.5)$ & \multirow{8}{*}{$<0.001$} \\
\hline Police & $51(30.4)$ & $2(1.3)$ & \\
\hline Minibus & $16(9.52)$ & $32(18.6)$ & \\
\hline Ambulance & $14(8.3)$ & $88(51.2)$ & \\
\hline Company car & $1(0.6)$ & 0 & \\
\hline Lorry & $3(0.6)$ & 0 & \\
\hline Walked & 0 & $2(1.3)$ & \\
\hline Missing & 2 & 1 & \\
\hline \multicolumn{4}{|c|}{ Time to presentation (hours), $\mathbf{n}(\%)$} \\
\hline $0-4$ & $144(86.2)$ & $81(47.4)$ & \multirow{8}{*}{$<0.001$} \\
\hline $5-8$ & $11(6.6)$ & $23(13.5)$ & \\
\hline $9-16$ & $6(3.6)$ & $17(9.9)$ & \\
\hline $17-24$ & $1(0.6)$ & $12(7.0)$ & \\
\hline $25-48$ & $3(1.8)$ & $18(10.5)$ & \\
\hline $49-96$ & 0 & $11(6.4)$ & \\
\hline$>96$ & $2(1.2)$ & $9(5.3)$ & \\
\hline Missing & 3 & 2 & \\
\hline \multicolumn{4}{|l|}{ Setting of injury, n (\%) } \\
\hline Road/street & $77(48.1)$ & $37(21.5)$ & \multirow{9}{*}{$<0.001$} \\
\hline Home & $58(36.3)$ & $124(72.1)$ & \\
\hline Public space & $2(1.25)$ & $2(1.2)$ & \\
\hline Work & $1(0.6)$ & $1(0.6)$ & \\
\hline School & $1(0.6)$ & $2(1.2)$ & \\
\hline Farm & $1(0.6)$ & $2(1.2)$ & \\
\hline Sports & $1(0.6)$ & $2(1.2)$ & \\
\hline Other & $19(11.9)$ & $2(1.2)$ & \\
\hline Missing & 10 & 1 & \\
\hline
\end{tabular}


performed in order to obtain a comprehensive understanding of paediatric trauma to implement appropriate interventions in LMICs.

Though all cause paediatric trauma mortality was dominated by head injuries, the preponderance of burn injury related mortality in the IHD cohort was not entirely surprising. The natural course of burn injury death is secondary to sepsis and multi-system organ failure. This cascade is delayed from initial injury and allows patients time to present to the hospital. Consequently, addressing paediatric burn mortality requires both burn prevention and improved management of initial resuscitation and sepsis. As previously described in a study by our group describing burn characteristics at $\mathrm{KCH}$, burn mortality is correlates reliably with burn size.

Comparing our IHD and PHD cohort data to other developing countries with minimal pre-hospital care reveals a similar distribution of injury mechanisms. However, other studies show a larger predominance of RTI and head trauma for both IHD and PHD and less mortality due to burns. In-hospital paediatric mortality data from a South African emergency department showed 55\% mortality for head trauma (48 of 88 ) with the second leading cause being thorax injuries (14 of 88$)$. However, this study did not include prehospital deaths and did not follow patients through their hospital course. In similar study in Tehran, $50 \%$ of paediatric mortality was attributed to RTIs, $18 \%$ from burns, $6 \%$ from falls, and $6 \%$ from poisoning. The majority of the RTI, poisoning, and drowning deaths occurred in the pre-hospital setting, while $92 \%$ of burn fatalities were IHD.

In comparison to data from developed countries, combined paediatric trauma PHD and IHD from Finland showed 85.6\% of fatal injuries were due to head trauma, $11 \%$ were due to penetrating wound including guns and stab wounds, and only $2.5 \%$ due to burns. This study was unique as all patients with sudden or traumatic death must be autopsied and this study included comprehensive PHD data. In contrast to data from Malawi, the majority of in-hospital death was due to severe brain injury, with very low incidence of paediatric burns. Paediatric IHD from Denmark showed majority of IHD were due to road traffic accidents $(54.2 \%)$, burns $(25 \%)$, and falls $(16.7 \%)$. This has improved correlation with Malawi's data, as burns compose a larger percentage of IHD.

In a study comparing trauma systems at three varying economic levels, pre-hospital death has been shown to inversely correlate with the maturity of the pre-hospital system. Mock et al. showed the region with the lowest economic status, Kumasi, Ghana, had the highest prehospital mortality at 51\%; Monterrey, Mexico had a $40 \%$ pre-hospital mortality; and at the highest economic status, Seattle, Washington had the lowest pre-hospital mortality at $21 \%$. Overall mortality decreased with the increase in economic status, with pre-hospital mortality being the main driver of the overall mortality decline. Significant differences between pre-hospital care were increased time to presentation in Ghana (102 $\mathrm{min} \pm 126 \mathrm{~min}$ ) compared to Seattle (31.1 min $\pm 9.8 \mathrm{~min}$ ), with $99.5 \%$ arriving in less than 1 hour; as well as, no pre-hospital care provided in Kumasi compared to advanced pre-hospital treatment in Seattle with airway and resuscitation management. Due to the lack of pre-hospital care and a mature trauma system, the majority of PHD that may have potentially survivable injuries do not arrive at a trauma center within the golden hour and may therefore die from potentially survivable injuries. The establishment of a pre-hospital trauma system in a developing country has the ability to decrease mortality up to $25 \%$. The findings of these studies are relevant to the planning of a trauma system in Malawi.

The transportation of the majority of pre-hospital deaths via private vehicle and police illustrates the lack of prehospital system in Malawi. If trauma occurs on the road, police are the first responders and often transport the most seriously injured trauma patients to the hospital. Successful pre-hospital trauma systems in the region have trained commercial drivers to provide care for motor vehicular trauma along with including basic trauma life support courses for first responders. Paediatric trauma management training for health care providers has also proven to improve mortality. Basic life support training for police and taxi cab drivers may substantially mitigate trauma mortality in Malawi.

The limitations of this study are those inherent to any study with a retrospective design. Our study included only paediatric traumas patients presenting to one tertiary care hospital. Not all paediatric deaths in this region were included, as some patients may have presented to lower tier hospitals and died from their injuries or were directly taken to the morgue and hence some selection and presentation bias exists. Furthermore, due to the limited resources that exist in Malawi, formal autopsies were not performed on any of the patients. Hence, the cause of death could only be inferred by clinical examinations only in the PHD patients and in the IHD based on clinical examination and hospital course.

\section{Conclusions}

Head injury is the main driver of overall paediatric trauma mortality in Malawi. However in the inpatient cohort, burn is the major of driver of paediatric mortality. Inclusion of pre-hospital data is imperative in the creation of any trauma surveillance program in order to capture and characterise the true magnitude and aetiology of injury in any environment. A robust pre-hospital program is an invaluable component of a trauma system, with its parts including an ambulance system, trained paramedic personnel, and a communication network. In the absence of a prehospital system, utilising police and public transportation providers trained in basic trauma life support to mitigate trauma death. Aggressive burn resuscitation for hospitalised patients and prevention of burn related sepsis may reduce may reduce burn mortality. Finally, a national strategy for primary injury prevention in Malawi is necessary to reduce both head injury and burn injury paediatric deaths.

\section{Competing interests}

All authors declare that they have no competing interests related to this work.

\section{References}

1. Lopez AD, Mathers CD, Ezzati M, Jamison DT, Murray CJ. Global and regional burden of disease and risk factors, 2001: systematic analysis of population health data. The Lancet 2006 Jun 2;367(9524):1747-57.

2. van As AS. Paediatric trauma care. Afr J Paediatr Surg 2010 Sep 1;7(3):129

3. Baingana FK, Bos ER. Changing patterns of disease and mortality in Sub-Saharan Africa: an overview. In Jamison DT, Reachem RG, Makgoba MW, Bos ER, Baingana FK, Hofman KJ, et al., editors. Disease and Mortality in Sub-Saharan Africa. 2nd edition. Washington, DC: World Bank Publications; 2006. 
4. Peden MM. World report on child injury prevention. World Health Organization; 2008.

5. Marson AC, Thomson JC. The influence of prehospital trauma care on motor vehicle crash mortality. J Trauma Acute Care Surg 2001 May 1;50(5):917-21.

6. Mock C, Nguyen S, Quansah R, Arreola-Risa C, Viradia R, Joshipura M. Evaluation of trauma care capabilities in four countries using the WHO-IATSIC Guidelines for Essential Trauma Care. World J Surg 2006 Jun 1;30(6):946-56.

7. Odero W, Garner P, Zwi A. Road traffic injuries in developing countries: a comprehensive review of epidemiological studies. Trop Med Int Health 1997 May 1;2(5):445-60.

8. Task Force on Community Preventive Services. Recommendations to reduce injuries to motor vehicle occupants: increasing child safety seat use, increasing safety belt use, and reducing alcohol-impaired driving. Am J of Prev Med 2001 Nov 30;21(4):16-22.

9. Qureshi JS, Ohm R, Rajala H, Mabedi C, Sadr-Azodi O, AndrénSandberg $\AA$, et al.. Head injury triage in a sub Saharan African urban population. Int J Surg 2013 Apr 30;11(3):265-9.

10. Ghajar J. Management of pediatric head injury. Pediatr Clin North Am 1992;39:1093.

11. Mock CN, Maier RV. Low utilization of formal medical services by injured persons in a developing nation: health service data underestimate the importance of trauma. J Trauma Acute Care Surg 1997 Mar 1;42(3):504-13.

12. Cooper A, Barlow B, Davidson L, Relethford J, O'Meara J, Mottley L. Epidemiology of pediatric trauma: importance of population-based statistics. J Pediatr Surg 1992 Feb 29;27(2):149-54.
13. Tyson AF, Boschini LP, Kiser MM, Samuel JC, Mjuweni SN, Cairns BA, Charles AG. Survival after burn in a sub-Saharan burn unit: Challenges and opportunities. Burns. 2013 Dec 31;39(8):1619-25.

14. Herbert HK, van As AB, Bachani AM, Mtambeka P, Stevens KA, Millar AJ, et al.. Patterns of pediatric injury in South Africa: an analysis of hospital data between 1997 and 2006. J Trauma Acute Care Surg 2012 Jul 1;73(1):168-74.

15. Roudsari BS, Shadman M, Ghodsi M. Childhood trauma fatality and resource allocation in injury control programs in a developing country. BMC Public Health 2006 May 2;6(1):1.

16. Souminen P, Kivioja A, Öhman J, Korpela R, Rintala R, Olkkola KT. Severe and fatal childhood trauma. Injury 1998 Jul 31;29(6):42530.

17. Do HQ, Steinmetz J, Rasmussen LS. In-hospital mortality pattern of severely injured children. Injury 2012 Dec 31;43(12):2060-4.

18. Mock CN, Jurkovich GJ, Arreola-Risa C, Maier RV. Trauma mortality patterns in three nations at different economic levels: implications for global trauma system development. J Trauma Acute Care Surg 1998 May 1;44(5):804-14.

19. Henry JA, Reingold AL. Prehospital trauma systems reduce mortality in developing countries: a systematic review and metaanalysis. J Trauma Acute Care Surg 2012 Jul 1;73(1):261-8.

20. Mock CN, Quansah R, Addae-Mensah L, Donkor P. The development of continuing education for trauma care in an African nation. Injury 2005 Jun 30;36(6):725-32. 